\title{
An Operational Drought Risk Management Framework Based on stream-flow Intelligent Internet control ${ }^{*}$
}

\author{
Rongfang Li \\ College of Information Sciences and Technology, Donghua University, Shanghai 201620, P. R. China \\ Jiangxi Provincial Institute of Water Sciences, Nanchang, Jiangxi 330000, P. R. China \\ Lijun Cheng ${ }^{1,2,4}$, Yongsheng Ding ${ }^{1,2^{*}}$, K. Khorasani ${ }^{4}$, Yunxiang Chen $^{3}$, and Wei Wang ${ }^{1,2}$ \\ 1. College of Information Sciences and Technology, Donghua University, Shanghai 201620, P. R. China \\ 2. Engineering Research Center of Digitized Textile \& Fashion Technology, Ministry of Education, \\ Donghua University, Shanghai 201620, P. R. China \\ 3. Jiangxi Provincial Institute of Water Sciences, Nanchang, Jiangxi 330000, P. R. China \\ ${ }^{4}$ Department of Electrical \& Computer Engineering, Concordia University, \\ Montreal, Quebec, H3G1M8 Canada
}

\begin{abstract}
In this paper, an operational drought risk management framework based on the stream-flow intelligent internet control is proposed. In the proposed framework drought can be predicted, evaluated and mitigated by using a dynamic stream-flow control under the sensors detection. The framework mainly includes four sequential steps: (i) the stream-flow prediction, (ii) the stream-flow deficit index (SDI) analysis, (iii) the drought multiple regions response, and (iv) the stream-flow balance control. In order to instantiate a specific framework management, intelligence methods are utilized in these processes, namely the generalized regression neural network (GRNN) algorithm for the stream-flow prediction and the collaborative particle swarm optimization (CPSO) for the reservoirs water collaborative operation. Finally, a specific case study corresponding to the Fu basin in China is investigated to test the operability and reliability of the proposed drought risk management.
\end{abstract}

Keywords: Drought risk assessment; Generalized regression neural network; Dynamic stream-flow prediction; Data-driven methods; Collaborative particle swarm optimization

*Corresponding author :Yongsheng Ding, PhD \& Professor, Email: ysding@dhu.edu.cn 


\section{Introduction}

Water scarcity has frequently occurred in recent years in the world. Numerous studies show that the condition is being further aggravated by the rising water demands of municipalities and industries, the growing population, and the gradual climate changes ${ }^{1,2}$. As a result, drought hydrology has been receiving much attention not only in research, but also in public lives ${ }^{3}$. Drought is a long period of meteorological anomaly characterized by extreme lack of precipitation, which causes significant damages in both natural environment and human life ${ }^{4,5}$. Consequently, it is imperative to construct an entity drought management system to explore the inherent laws and manage the risk holistically like a business in order to mitigate the impacts of drought on water resource systems.

Stream-flow observations collected from near-natural catchments are of paramount importance for water detection and water attribution studies. Any organizational regulation actions for the stream-flow has a direct impact on the lives of the residents, the industrial production, the agricultural irrigation as well as the adaptation and policy options ${ }^{6}$. The drought management is fully integrated into the stream-flow development efforts, and the manner to control the stream-flow at different lead times often is a first step ${ }^{7}$. Such a processing with a stream-flow drought management is often referred to as a drought risk management, which can ensure the development process for risks of short-term climate variability and long-term water flow changes ${ }^{6,8}$. In recent years, much effort has been made in the drought prediction, preparedness, adaptation and mitigation, such as the stream-flow drought time series forecasting ${ }^{5,9,10}$, the real-time drought forecasting in multi-reservoir operations ${ }^{11}$, simulating time series of climate variables for drought management ${ }^{12}$, etc. All of the above works have detected an aspect of the drought risk by certain spatial variability. The drought risk management has been dispersed at several separable portions sets. However, risk management, as is known as a consecutive procedure which includes risk monitoring, risk identification, risk evaluation and sequential decision-making. Each of the sessions depends on the previous stage and has a mutual interaction with the other parts. If they are separated into pieces for investigation, it becomes difficult to understand, apply, evaluate and trust risk management capabilities ${ }^{12,} 13$. Therefore, there is a need for a comprehensive drought risk management system.

In the integrated internal control-risk management (IICRM) framework that is borrowed from business risk management issued by COSO in 2004 14, 15, "Internal Control" is involved in and is an inseparable part of the risk management system. The risk management is no longer only concerned with the prediction of the risk occurrence in each segment, but is also concerned with the analysis of the risk with certain related portions that are all properly integrated. With the advent of internet, more objects are becoming embedded within wireless sensors and are gaining the ability to communicate in the Internet ${ }^{16}$. To implement a quantitative drought risk management system, one should consider how to use either discrete or continuous measurement systems to understand the operational risk across each of the drought risk prediction, evaluation, decision and regulatory processes and steps.

In this paper, an automated and fully data driven drought risk and integrity management system embedded in the Internet is proposed which is derived from IICRM. In the proposed framework, the drought variable parameters including the rainfall, the streamflow, and the reservoir can be automatically detected and recorded by a variety of sensors, and then a datadriven empirical modeling technique ${ }^{17,18}$ is used to evaluate the stream-flow as a drought risk degree. Finally, a dynamic collaborative model attempts to control all the reservoirs to discharge or bump and achieve a dynamic stream-flow balance in different regions. Through a specific case application in the Fuhe basin in China, it is confirmed that the risk management mode of the drought can improve water control processes and reduce the drought prediction costs effectively.

The main contributions of this paper are as follows: (i) An operational framework for the drought risk management is proposed which is based on the datadriven intelligent Internet control;

(ii) In the proposed framework, the drought can be measured, evaluated and mitigated by using a dynamic stream-flow adjusting in a life cycle of risk management with intelligent system models, which includes the generalized regression neural network (GRNN), drought stream-flow deficit index (SDI) risk identification 
algorithm and the collaborative particle swarm optimization (CPSO);

(iii) A case scenario is used to clarify and verify the framework applicability and operability in the streamflow drought risk management.

The paper is organized as follows. The operational drought risk management framework which is based on data-driven intelligent Internet control is presented in Section 2. The basic intelligent research methodology is reviewed in Section 3. The details of the drought risk management system based on the dynamic stream-flow management and analysis are illustrated in Section 4. A case study scenario in the Fuhe basin in China is examined through the proposed framework in Section 5. Finally, concluding remarks and further works are provided in Section 6.

\section{A Data-Driven Operational Drought Risk Management Framework}

A dynamic drought risk management system is presented according to a simple chronological order in Fig. 1 Part 1, which is referred to as an operational drought risk management of the Internet control (ODRMIC). The operational drought risk management framework based on the data-driven intelligent Internet control consists of seven interrelated steps that are shown in Fig. 1 Part 1.

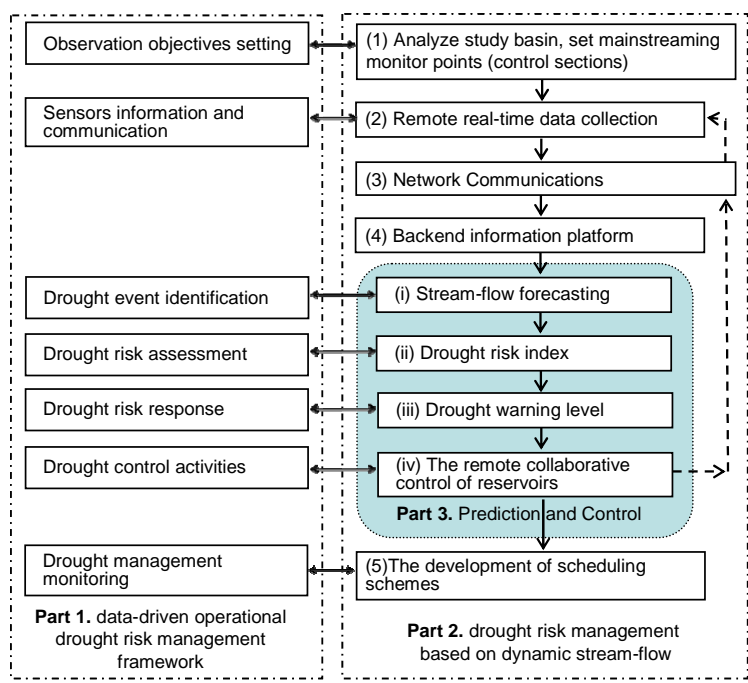

Fig. 1. The operational drought risk management framework based on the data-driven intelligent Internet control It should be emphasized that the framework is based on the assumed conditions in the study that the regional stakeholders have established the corresponding proportion of the watershed for coordinating water under the water shortage scenarios. They will provide the support to the coordination group member organizations in assuming their agreed upon roles and responsibilities under the drought situation. The drought risk management system is derived from the manner the management runs an enterprise and is integrated with the management process ${ }^{19}$. These components are as follows:

(i) Setting observable objectives - Before drought risk management systems, the studied region would require a careful analysis, and then the observable objectives (known also as control sections) are set, that include the steam-flow monitor points, the reservoirs dam monitor points, and the rainfall hydrometric station monitor points etc. These monitoring points will be used to collect the data to identify the potential drought events.

(ii) Sensors information and communication - The sensors information are identified, captured, and communicated in a time slice form under different sensors from the monitoring points. Effective communication also occurs in a broader sense, flowing down, across, and up to the backend information platform through the network communications.

(iii) Drought event identification - Based on the sensors information, certain methods are used to forecast the future stream-flow, and the predicting results will be channelled back to a drought management's strategy or the drought plan-setting processes.

(iv) Drought risk assessment - Risks are analyzed through a drought risk index calculation. The index values will impact and then determine the drought warning range of the regions. Drought events may be classified according to the index deficit severity.

(v) Drought risk response - Management system can select responses - avoiding, accepting, mitigating, or sharing risk according to the value of the drought severity assessment as performed in step (iv). For a short-term drought, if the drought can be mitigated through controlling the gate opening of the reservoir dam - then activate the remote reservoirs collaborative control process, or else develop a set of actions to align risks with the region government's risk tolerances as well as the risk response measures.

(vi) Drought control activities - To modify the drought risk, start remote collaborative control of the reservoirs and then drain the water in the control. However, the 
uncontrolled long-term drought, policies and procedures need be established and implemented to help ensure the risk responses for an effective execution.

(vii) Drought management monitoring - When the system performs the reservoirs dispatching water scheduling schemes, the sensors can monitor in realtime all the monitoring points of the water variance until each monitoring point satisfies the minimum required water, so that the reservoirs drainage action will then stop.

Drought risk management is an iterative and sequential process in which almost any component can and does influence another one, but it is not strictly performing each process. In the following section, we will instantiate a specific case to explain the drought event identification, the drought risk assessment, the drought risk response and the drought control activities using an intelligent operational method, which corresponds to the Fig. 1 part 2.

\section{Drought Risk Management Based on Dynamic Stream-Flow Management and Analysis}

The stream-flow is the volume of water that moves on a designated point over a fixed period of time ${ }^{20}$. The flow of a stream is directly related to the amount of water moving off the watershed into the stream channel. It is affected by the weather conditions, whereas it is increasing during the rainstorms and decreasing during the dry periods. On the other hand, it changes with the seasons of the year; decreases during the summer months and reaches the lowest flow for most streams and rivers in most of the country in the months of August and September ${ }^{10}$. In the following sections, the time series stream-flow analysis is represented as the drought behavior and is researched under different periods. There are a number of other factors for drought detection, such as the nature of the soil moisture deficit and the groundwater deficit or the precipitation deficit, etc. The deficits indices are only a part of the droughts measure in any specific application. They are included in this framework as a specific illustrative case study. Due to space limitations, the readers can refer to the multi-index drought prediction for more details.

Fig. 1 Part 2 shows a specific stream-flow case to explain the drought risk management process. The blue box sections in Fig. 1 Part 3 are the refinements of the entire process, and they will be elaborated in detail in the following sections. Fig. 2 shows the refinement details of the steam-flow implementation analysis for the drought risk management by using data-driven intelligence methods. In Fig. 2, each counterpart of Fig. 1 Part 2 has been marked. All of these processes in Fig. 2 are performed in sequence as follows.

(i) Monitor the real-time stream-flow, the rainfall and the reservoir water level in each control section.

(ii) The GRNN model is established to forecast the short-term and the long-term stream-flow based on the historical and real-time sensor data, respectively. The detail model construction is illustrated in Section 3.1.

(iii) A stream-flow deficit index (SDI) is calculated as a drought evaluation standard for measuring the degree and level of the water resource shortage. Through the $S D I$, drought-warning level in different regions is obtained and it will yield a drought warning level, corresponding to a drought response degree. The details on the calculations are illustrated in Section 3.2.

(iv) The reservoirs control system will decide whether to perform the water dispatching or not as well as which reservoirs need to be discharged with the drought degree levels established in step (iii).

(v) When the drought degree reaches or exceeds the early drought warning grade and level, a real-time water regulation is initiated to perform by the CPSO in parallel. CPSO will deploy the water in each reservoir and decide which one need to drain and how long the reservoirs drainage last, the details on the process of the overall water allocation is illustrated in Section 3.3.

(vi) The stream-flow remote sensing device can detect water variation in every control section and renews these real-time data into the backend information platform.

(vii) When the real-time stream-flow of the control sections in the information platform satisfies the minimum water usage, then the reservoirs discharging stops.

(viii) Output the final water dispatch scheduling schemes to the water managers as well as the impacted water departments. 


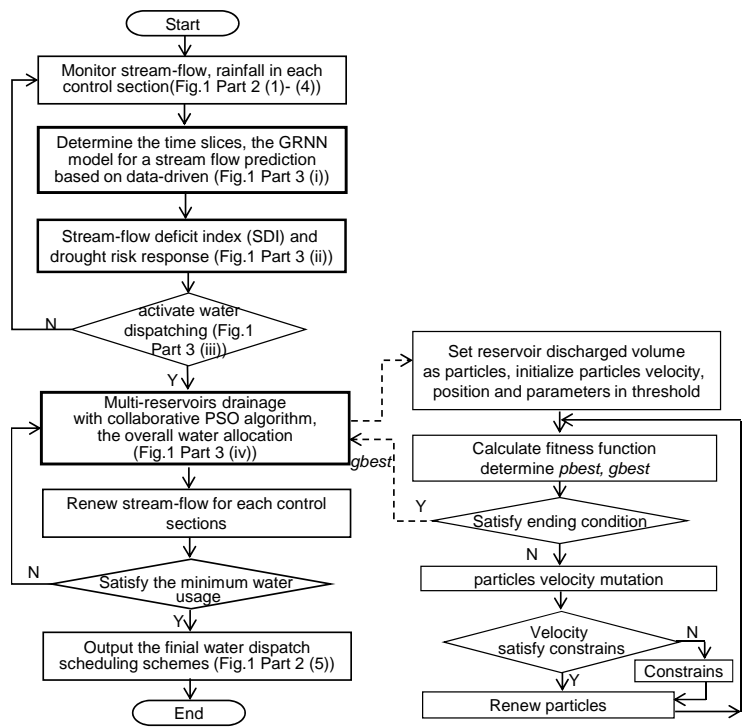

Fig. 2. A detailed process of the data-driven steam-flow control for drought control.

In the following, we are going to describe in detail the key steps that are described under items (ii), (iii), (v).

\subsection{Data-driven stream-flow prediction model}

The stream-flow is relevant to hydrological and meteorological problems and tasks. By integrating the long-term historical data with the real-time collected data in every hydrological station a time series analysis can be performed in order to detect the drought risk in future several days or a month. Many data-driven models ${ }^{21}$, including linear autoregressive (AR) ${ }^{22}$, nonparametric or nonlinear chaos-based neural networks (NN) ${ }^{23}$, have made great progress in hydrologic time series prediction in the past decades. GRNN algorithm is a typical chaos-based forecast method for dynamic time series data ${ }^{24}$. It uses a non-parametric probability density estimation to approximate the underlying regression surface of the training data available. For this reason it is also called the probabilistic neural networks.

Given a dataset $T$ having $n$ examples, $T=\left\{\left(x_{1}, y_{1}\right),\left(x_{2}, y_{2}\right), \ldots,\left(x_{n}, y_{n}\right), x_{i} \in R_{n}, y_{i} \in R \quad\right.$, $i=1,2, \ldots, n\}$, where $x_{i}$ is an input variable and $y_{i}$ is the desired target. Let us define the Euclidean distance $D_{i}$, which is used as a measure of how well the training sample $x_{i}$ can represent the point position of prediction input $x$. The estimate $\hat{y}(x)$ can be visualized as a weighted average of all of the observed value $y_{i}$, where each observed value is weighted exponentially according to its Euclidean distance from $x{ }^{24}$, that is:

$$
\hat{y}(x)=\frac{\sum_{i=1}^{n} y_{i} \exp \left(-\left\|D_{i}\right\|^{2} / 2 \delta^{2}\right)}{\sum_{i=1}^{n} \exp \left(-\left\|D_{i}\right\|^{2} / 2 \delta^{2}\right)}
$$

where $\left\|D_{i}\right\|^{2}=\left(x-x_{i}\right)^{\mathrm{T}}\left(x-x_{i}\right)$, and $\delta$ is the square deviation, also known as the smoothing parameter.

GRNN functions through the four-layers, namely: input layer, hidden layer, summation layer and output layer. The input layer only passes the input vector to the RBF hidden layer; in the hidden layer, the calculation performed in each pattern neuron is $\exp \left(-\left\|D_{i}\right\|^{2} / 2 \delta^{2}\right)$, with a normal distribution centers at each training sample. In the summation layer, the signals of the $i$-th pattern neuron, applied to the denominator neuron are weighted with the corresponding values of the training samples $y_{i}$. The weights on the signals applied to the numerator neuron are one. In the output layer, the output is simply a weighted average of the target values of the training cases close to the given input case as given by the expression in Eq.(1).

\subsection{Drought risk identification and evaluation}

Drought is generally considered as periods with insignificant water resources for sustaining activities of a region. There are a variety of indices to definite the drought degrees, generally the most adopted degree of drought is what the American Meteorological Society has proposed in $2004{ }^{25}$, namely the degree of the water deficit. In the present study, the stream-flow deficit index (SDI) is used as an index to evaluate the drought degree. $S D I$ is statistically similar to most other commonly used Standard Precipitation Index (SPI) ${ }^{26}$, which is based on the basic method used to derive drought events from continuous or discrete records of precipitation. The SDI is calculated by scaling the cumulative stream-flow values by using the median, the maximum, and the below minimum cumulative values as expressed in Eqn. (2)-(3). It overcomes the problem of fitting a statistical distribution to the cumulative stream-flow data ${ }^{30}$, specifically,

If $S W_{i, j} \leq M S W_{j}, S D I_{i, j}=-\frac{S W_{i, j}-M S W_{j}}{M S W_{j}-\min . S W_{j}} \times 100(2)$ 
If $S W_{i, j}>M S W_{j}, S D I_{i, j}=\frac{S W_{i, j}-M S W_{j}}{\max . S W_{j}-M S W_{j}} \times 100$

where $i$ denotes the years, $j$ denotes the day of the year (1 to 365$), S D I_{i, j}$ denotes the stream-flow deficit index (\%), $S W_{i, j}$ denotes the cumulative stream-flow for the period of interest $(\mathrm{mm}), M S W_{j}$ denotes the long-term median cumulative stream-flow for the period of interest $(\mathrm{mm})$, max.SW $W_{j}$ denotes the long-term maximum cumulative stream-flow for period of interest $(\mathrm{mm})$, and $\min . S W_{j}$ is the long-term minimum cumulative stream-flow for period of interest $(\mathrm{mm})$. These equations are used for scaling in order to create an index which has a lower bound of -100 and an upper bound of +100 .

\subsection{Optimal allocation of water by reserves dispatching}

The stream-flow can be divided into the two parts, the main-streaming and the tributary. The water shortage in the tributary part can pass its information to the main-streaming level by level so that ultimately the information will be fed back to its control reservoir. The reservoirs in different study areas cooperate and coordinate their activities to maintain a water balance and the stream-flow obtains the overall water allocation. The behavior of the reservoir is known as a collaborative optimal scheduling of the multi-level water.

The particle swarm optimization (PSO) algorithm, which is derived from the cooperative behavior of decentralized, self-organized systems ${ }^{27}$, is an evolutionary computational technique ${ }^{31}$. It has been widely applied to multivariate function optimization problems. Here, the collaborative PSO (CPSO) algorithm is used to determine in parallel the collaborative optimal water discharging in different reservoirs in multi-regions. Due to space limitations the reader can refer to the literature 28 and 29 for detailed processes and usage of this algorithm.

We assume that the reservoir outflows are represented as particles and the solution hyperspace dimensionality $D$ of each reservoir is associated with the dispatch period $t$. Given that for scheduling the time is 12 months a year, with month as a dispatching unit, then $D$ is set to 12 and for day as a dispatching unit, then $D$ is set to 365 . The particle $Q_{i}$ at the $i$-th iteration is defined according to

$$
Q_{i}=\left(q_{i 1}^{1}, q_{i 1}^{2}, \cdots, q_{i 1}^{D}, q_{i 2}^{1}, q_{i 2}^{2}, \cdots, q_{i 2}^{D}, \cdots, q_{i k}^{1}, q_{i k}^{2}, \cdots, q_{i k}^{D}\right)
$$

where $q_{i j}^{t}$ represents the $j$-th reservoir discharge at the $t$ th time corresponding to the $i$-th iteration, and $k$ denotes the number of reservoirs. The particle velocity vector $V_{i}$ defines the velocity change rate of the reservoir discharge corresponding to the $i$-th iteration, and the velocity of each particle represents the transition rate of every reservoir outflow according to

$$
V_{i}=\left(v_{i 1}^{1}, v_{i 1}^{2}, \cdots, v_{i 1}^{D}, v_{i 2}^{1}, v_{i 2}^{2}, \cdots, v_{i 2}^{D}, \cdots, v_{i k}^{1}, v_{i k}^{2}, \cdots, v_{i k}^{D}\right)(5)
$$

In practice, the velocity rate of the reservoir discharge is directly proportional to the reservoir storage water capacity. Hence, in the reservoir discharged process, the reservoir storage water always becomes smaller and smaller than the previous time. This will cause the reservoir flow rate to have different variation ranges in different times. In this case, the reservoir outflow capability has a variable scope. The minimum and maximum outflow velocities can be calculated according to the following equations, respectively, corresponding to the $t$-th period,

$$
\begin{aligned}
& v_{i \text { min }}^{t}=\alpha q_{i \text { min }}^{\mathrm{t}}+(1-\alpha) q_{i \text { max }}^{\mathrm{t}} \\
& v_{i \text { max }}^{t}=\beta q_{i \text { min }}^{\mathrm{t}}+(1-\beta) q_{i \text { max }}^{\mathrm{t}}
\end{aligned}
$$

where $q_{i \max }^{\mathrm{t}}$ denotes the maximum reservoir outflow capability of the $i$-th reservoir, $q_{i \min }^{\mathrm{t}}$ denotes the minimal reservoir outflow in $t$-th period, $\alpha=(1+\operatorname{rand}()) / 2, \beta=(1-\operatorname{rand}()) / 2,1 \leq j \leq N$, $1 \leq t \leq D$, and rand() is a random variable. The collaborative optimal water discharge in different reservoirs in multi regions is given as follows, which is shown in the right part of Fig.2.:

Step 1: Initialize $n$ particles in the population $U=\left\{P_{1}, P_{2}, \ldots, P_{i}, \ldots, P_{n}\right\}$, set the parameters in the PSO, namely the particle population size, the solution hyperspace dimensionality, and the maximum number of iterations;

Step 2: Calculate the values of the fitness function which can be defined as the minimum shortage of the water storage in each control section:

$$
\text { fitness }=\min \sum_{s}\left[D_{s}(t)-R_{s}(t)\right]
$$

where $R_{s}(t), D_{s}(t)$ denote the $s$-th control section's water supply capacity (the recommended reservoir outflow) and water demand capacity, respectively. The goal is to have the particles move towards a small fitness direction.

Step 3: Select the particle position with the lowest fitness value that is observed in all particles in a certain generation as the best overall position (gbest ) $P_{g d}$ and the smallest fitness value in each generation as the best current generation position ( pbest ) $P_{b d}$; 
Step 4: Check the termination condition. If the maximum number of iterations has been reached or the required minimal fitness error accuracy has been obtained,then the best individual is obtained in the current population, output the ( gbest ) $P_{g d}$ and terminate the process; otherwise, go to the next step;

Step 5: Perform the particle velocity mutation using Eq. (9) :

$$
V_{i}^{\text {iter }+1}=w V_{i}^{\text {iter }}+c_{1} r_{1}^{\text {iter }}\left(p_{\text {ibest }}^{\text {iter }}-Q_{i}^{\text {iter }}\right)+c_{2} r_{2}^{\text {iter }}\left(g_{\text {best }}^{\text {iter }}-Q_{i}^{\text {iter }}\right)
$$

where iter denotes the iteration time; $1 \leq i \leq n ; c_{1}$ and $c_{2}$ are the acceleration coefficients, that are commonly set to $2 ; r_{1}^{\text {iter }}$ and $r_{2}^{\text {iter }}$ denote two random variables in the range $[0,1]$; and $w=\frac{1}{\text { iter }}$ denotes an inertia weight whose values decline linearly over iter .

Step 6: Set the velocity in its maximum or minimum value range if it exceeds the velocity limitation given Eqn. (6) and (7).

Step 7: Update particle's position vectors by using Eq. (10), then return to Step 2.

$$
Q_{i}^{i t e r+1}=Q_{i}^{i t e r}+V_{i}^{i t e r+1}
$$

\section{A Case Study}

\subsection{Study area}

The Fuhe basin that is located in the Jiangxi province of China has an area of $15,811 \mathrm{~km}^{2}$ and is covered with significant agricultural fields. The Fu river is its main water supply source which originates from the South Guangchang city and ends in the North Poyang Lake, covering a total length of $312 \mathrm{~km}$, with a yearly average flow of $483 \mathrm{~m}^{3} / \mathrm{s}$. The basin has much rainfall every year. However, it suffers from the subtropical high pressure every year, and the spatial and the temporal distribution of water resources are extremely uneven. The yearly mean of precipitation is $838 \mathrm{~mm}$ from April to June, accounting for $48 \%$ of the annual precipitation. However, during the agricultural peak months of July to September, it only has $329 \mathrm{~mm}$ of precipitation, which accounts for only $17.5 \%$ of the annual precipitation. During the period 2003-2009, thousands of people had to face with the drinking water shortages in that period. In order to have an appropriate arrangement usage of the water, a drought risk forecast and water resource management are drawn up in advance so that governments have a good measurement plan to confront with the upcoming water usage contradictions.
Data of the rainfall and the stream-flow from 1970 to 2008 are collected from 9 hydrometric stations (these are also caller control sections) and the data of the water level and tumble flow are collected in two big reservoirs. Fig. 3 shows the network schematic diagram of the Fuhe basin. The control sections, the large reservoirs and the main water usage regions are marked in the diagram. At the same time, the control sections as detection points will collect real-time runoff (steamflow) and precipitation data from the sensors, which lie in the key points of the river. The yearly statistical properties of the steam-flow of each control sections are shown in Table 1. It can be seen that there exist uneven water distributions in different regions in different seasons.

\subsection{Results and discussion}

When the data of the stream-flow sensors is entered into the information database, the GRNN will predict the stream-flow variation and determines whether the control section has a lack of water or not according to its SDI degree. If one of the control sections detects a water shortage, it will send the information to the

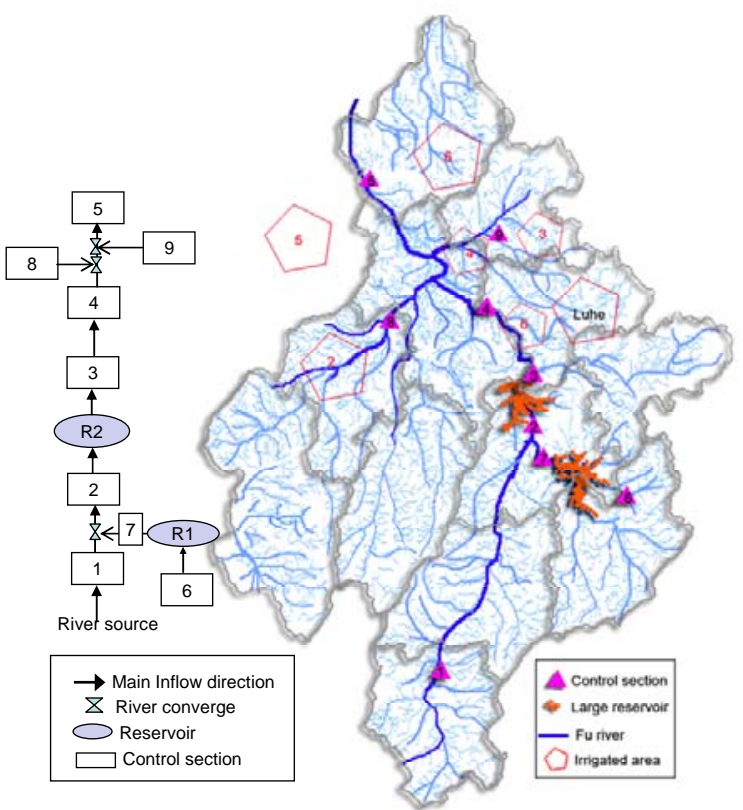

Fig. 3. The schematic distribution of the control sections, the large reservoirs and the main water usage regions in the Fuhe basin. 
Table 1. Statistical properties of control sections for the annual average runoff and rainfall series in the Fu Basin (1970-2008)

\begin{tabular}{llllllllll}
\hline \multirow{2}{*}{$\begin{array}{l}\text { Control } \\
\text { NO }\end{array}$} & sections & $\begin{array}{l}\text { Annual average stream-flow series } \\
\left(\mathrm{m}^{3} / \mathrm{s}\right)\end{array}$ & $\begin{array}{l}\text { Max } \\
\left(\mathrm{m}^{3} / \mathrm{s}\right)\end{array}$ & $\begin{array}{l}\text { Mean } \\
\left(\mathrm{m}^{3} / \mathrm{s}\right)\end{array}$ & $\begin{array}{l}\text { Standard } \\
\text { deviation }\end{array}$ & $\begin{array}{l}\text { Min } \\
(\mathrm{mm})\end{array}$ & $\begin{array}{l}\text { Max } \\
(\mathrm{mm})\end{array}$ & $\begin{array}{l}\text { Mean } \\
(\mathrm{mm})\end{array}$ & $\begin{array}{l}\text { Standard } \\
\text { deviation }\end{array}$ \\
\hline 1 & Shaziling & 5.3748 & 496.1733 & 103.1576 & 78.1151 & 3.4822 & 4.3126 & 6.20 & 2.0645 \\
\hline 2 & Nancheng & 2.0258 & 423.66 & 33.8196 & 22.9884 & 3.6561 & 4.5747 & 7.1258 & 2.0709 \\
\hline 3 & Miaofang & 2.3676 & 260.9882 & 39.8958 & 28.0788 & 3.1025 & 4.0379 & 4.2709 & 1.9535 \\
\hline 4 & Miaojiawan & 1.4154 & 127.8235 & 23.5434 & 14.6113 & 3.6024 & 4.6659 & 7.720 & 2.0581 \\
\hline 5 & Lijiadu & 0 & 44.2049 & 2.9765 & 1.5482 & 3.4132 & 4.3996 & 4.7741 & 1.8387 \\
\hline 6 & Lichuan & 0 & 325.17 & 64.0218 & 35.6321 & 4.0577 & 5.0091 & 7.27 & 2.4096 \\
\hline 7 & Hongmen & 0.3451 & 100.3733 & 7.3417 & 4.6551 & 3.7376 & 4.6337 & 6.8133 & 1.5903 \\
\hline 8 & Loujiacun & 0.5251 & 207.1633 & 16.9233 & 9.3239 & 3.4835 & 4.7675 & 7.7036 & 2.06 \\
\hline 9 & Mawei & 0.1004 & 560 & 31.9848 & 19.1619 & 3.8131 & 4.9411 & 7.6 & 2.4484 \\
\hline Average & 0 & 560 & 28.9251 & 49.5302 & 3.6437 & 3.7852 & 9.36 & 1.9865 \\
\hline
\end{tabular}

Table 2. The water dispatch proportion for each control section

\begin{tabular}{llllllllll}
\hline Station & No.1 & No. 2 & No. 3 & No. 4 & No. 5 & No. 6 & No. 7 & No. 8 & No. 9 \\
Proportion & $4.78 \%$ & $0.60 \%$ & $10.44 \%$ & $16.64 \%$ & $5.33 \%$ & $14.79 \%$ & $16.00 \%$ & $12.11 \%$ & $8.66 \%$ \\
\hline
\end{tabular}

Table 3. The reservoir storage outflow for the water dispatching in different months in 2008 (unit: $\mathrm{m}^{3} / \mathrm{s}$ )

\begin{tabular}{cccccccccccccc}
\hline Reservoir & Jan. & Feb. & Mar. & Apr. & May & June & July & Aug. & Sept. & Oct. & Nov. & Dec. \\
\hline R1 & 12.57 & 18.69 & 21.63 & 35.02 & 97.42 & 355.80 & 479.07 & 189.78 & 210.74 & 92.56 & 126.15 & 14.028 \\
R2 & 51.00 & 68.24 & 87.93 & 91.21 & 141.46 & 459.10 & 437.65 & 284.85 & 136.53 & 77.72 & 68.73 & 42.40 \\
\hline
\end{tabular}

Table 4. The cooperative results of the water dispatch proportion for the entire control sections

\begin{tabular}{ccccccccccccc}
\hline $\begin{array}{c}\text { Control } \\
\text { sections }\end{array}$ & Jan. & Feb. & Mar. & Apr. & May & June & July & Aug. & Sept. & Oct. & Nov. & Dec. \\
\hline No.1 & $4.4 \%$ & $4.3 \%$ & $4.3 \%$ & $4.8 \%$ & $4.6 \%$ & $4.5 \%$ & $4.6 \%$ & $4.6 \%$ & $4.7 \%$ & $4.4 \%$ & $4.5 \%$ & $4.2 \%$ \\
No.2 & $0.5 \%$ & $0.5 \%$ & $0.5 \%$ & $0.6 \%$ & $0.6 \%$ & $0.6 \%$ & $0.5 \%$ & $0.5 \%$ & $0.6 \%$ & $0.5 \%$ & $0.5 \%$ & $0.6 \%$ \\
No.3 & $10.2 \%$ & $9.9 \%$ & $10.1 \%$ & $10.4 \%$ & $10.4 \%$ & $10.5 \%$ & $9.4 \%$ & $10.1 \%$ & $10.3 \%$ & $10.1 \%$ & $10.2 \%$ & $9.6 \%$ \\
No.4 & $14.6 \%$ & $15.1 \%$ & $16.3 \%$ & $15.4 \%$ & $16.6 \%$ & $16.7 \%$ & $16.4 \%$ & $16.2 \%$ & $15.9 \%$ & $16.3 \%$ & $16.4 \%$ & $15.5 \%$ \\
No.5 & $4.8 \%$ & $4.8 \%$ & $5.0 \%$ & $5.0 \%$ & $5.3 \%$ & $5.1 \%$ & $5.3 \%$ & $4.9 \%$ & $4.8 \%$ & $5.0 \%$ & $4.7 \%$ & $4.2 \%$ \\
No.6 & $13.7 \%$ & $14.3 \%$ & $14.5 \%$ & $12.5 \%$ & $14.8 \%$ & $14.5 \%$ & $14.5 \%$ & $14.6 \%$ & $14.5 \%$ & $14.3 \%$ & $14.6 \%$ & $14.0 \%$ \\
No.7 & $14.1 \%$ & $15.4 \%$ & $15.4 \%$ & $13.5 \%$ & $16.0 \%$ & $16.1 \%$ & $15.7 \%$ & $15.3 \%$ & $15.9 \%$ & $15.9 \%$ & $15.8 \%$ & $15.8 \%$ \\
No.8 & $10.1 \%$ & $11.4 \%$ & $9.7 \%$ & $9.5 \%$ & $12.1 \%$ & $12.1 \%$ & $11.7 \%$ & $12.1 \%$ & $11.7 \%$ & $11.9 \%$ & $12.1 \%$ & $10.5 \%$ \\
No.9 & $8.2 \%$ & $8.4 \%$ & $8.5 \%$ & $7.6 \%$ & $8.7 \%$ & $8.5 \%$ & $8.5 \%$ & $7.8 \%$ & $8.0 \%$ & $8.3 \%$ & $8.3 \%$ & $7.4 \%$ \\
\hline
\end{tabular}


reservoir controlling system. After the discharging instruction is accepted and agreed upon, a dispatch water plan is initiated using the CPSO algorithm by adjusting the outflow of each reservoir. It will optimize the water volume for every stream segment to avoid a water shortage.

In the following, we simulate a cooperative result of the water dispatch proportion in a month. The primary parameters of the CPSO algorithm are set as follows: $D=12$ (month), $m=50$, iter ${ }_{\max }=1000$, $w_{\text {max }}=0.9, w_{\text {min }}=0.4, c_{1}=c_{2}=2$, where $D$ represents the solution hyperspace's dimensions; $m$ denotes the particle size of population; iter $_{\max }$ denotes the maximum iterations; $w_{\max }$ and $w_{\min }$ denote respectively the maximum and the minimum inertia weight; and $c_{1}$ and $c_{2}$ denote learning factors. The maximum and the minimum particle velocities can be calculated according to Eq. (6) and Eq. (7), respectively.

Table 2 shows the corresponding proportion of the watershed for coordinating the water during its shortage which is established by the study regional stakeholders. Table 3 shows the computational results of the reservoir storage outflow operation for the drought mitigation by using the CPSO algorithm for the next anticipated month. It can be seen that the reservoir storage outflow from the months of June to September are larger than the other months.

Table 4 shows the simulations for the water dispatch proportion of each control section after dispatching water for the next anticipated month. Comparing Table 2 with Table 4, the overall results in Table 4 are almost close to those in Table 2. Furthermore, the dispatching results have reached the water balance in different regions and have met the different region water demand, especially in the agricultural water requirement peaks from the months of June to September.

\section{Conclusions and Further Work}

With the advent of telecommunication revolution, it is a huge challenge for decision-makers to reconsider the drought risk management in the Internet environment. In this paper, we have proposed an operational drought risk management framework based on data-driven and intelligent Internet-based control solutions. The framework that is outlined addresses the drought identifying risk, the drought early warning system, the drought dynamic response and the drought mitigation solutions by means of management concepts. It transforms the new idea of applying an automated, consistent, data-driven methodology to ensure that the drought risk management is effective and autonomous. By utilizing the proposed management system, the drought mitigation becomes simple and operational by handling the captured dynamic data. In addition, water administrators will have better information on not just deciding on the current dry state, but also easily forecasting the anticipated drought in future times with confidence.

For the drought risk management framework, a specific stream-flow management and analysis is developed and presented in detail for each part of the drought risk management process. Through the use of the generalized regression neural network (GRNN) the stream-flow forecast for the drought event identification is achieved; by using the stream-flow deficit index (SDI) analysis in different monitoring points, the drought risk assessment is obtained, and finally, the remote collaborative control of reservoirs using the collaborative particle swarm optimization (CPSO) algorithm is implemented. The case scenarios corresponding to the Fuhe basin in China illustrates that the drought risk management is feasible and operational, and has a potential benefit for many thousands of households and livestock in the Fuhe basin.

The framework for the drought risk management is easy to operate, and the alternative implemented models are feasible in managing both short-term as well as long-term drought processes in another territory even when the available input datasets is different.

\section{Acknowledgements}

This work was supported in part by the Key Project of the National Nature Science Foundation of China (No. 61134009), the National Nature Science Foundation of China (No. 60975059), Specialized Research Fund for the Doctoral Program of Higher Education from Ministry of Education of China (No. 20090075110002), Specialized Research Fund for Shanghai Leading Talents, Project of the Shanghai Committee of Science and Technology (Nos. 11XD1400100, 11JC1400200, 10JC1400200), and the Fundamental Research Funds for the Central Universities. 


\section{References}

1. A. K. Mishra, V. P. Singh, V. R. Desai, Drought characterization: a probabilistic approach, Stoch Environ Res Risk Assess, 23 (2009): 41-55.

2. B. C. Bates, Z. W. Kundzewicz, S. Wu and S. Palutik Eds., Climate change and water, Intergovernmental Panel on Climate Change Secretariat, (IPCC, Geneva, 2008): 210.

3. K. M. Ashok and P. S. Vijay, Drought modeling - a review, Journal of Hydrology, (403) (2011): 157-175.

4. Jr. R. R. Heim, Drought indices: A review, Chapter 11. In Drought: A Global Assessment (Donald A. Wilhite, ed. volume 1), (Routledge: London, 2000) ,159-167.

5. A. K. Mishra and V. P. Singh, A review of drought concepts. J. Hydrol. 391(1-2) (2010): 202-216.

6. United Nations Development Programme Drylands Development Centre United Nations, Mainstreaming Drought Risk Management- a primer, UNDP 2011: 7-11.

7. C. Fernández, J. A. Vega, T. Fonturbel and E. Jiménez, Streamflow drought time series forecasting: a case study in a small watershed in North West Spain. Stoch. Environ. Res. Risk Assess, 23(2009): 1063-1070.

8. K. Stahl, H. Hisdal, J. Hannaford, L. M. Tallaksen, H. A. J. van Lanen, E. Sauquet, S. Demuth, M. Fendekova, and J. Jódar, Streamflow trends in Europe: evidence from a dataset of near-natural catchments, Hydrol. Earth Syst. Sci. Discuss., 7 (2010): 5769-5804.

9. C. L. Wu, K. W. Chau, Data-driven models for monthly streamflow time series prediction, Engineering Applications of Artificial Intelligence 23 (2010): 13501367.

10. R. Modarres, Stream-flow drought time series forecasting, Stoch Environ Res Risk Assess 21 (2007): 223-233.

11. W. C. Huang and C. C. Chou, Drought early warning system in reservoir operation: theory and practice, Water Resources Research, 41 (2005): 12.

12. A. Steinemann, Using climate forecasts for drought management. J. Appl. Meteorol. Climatol, 75 (2006): 1353-1361.

13. J. G. Carbone and K. Dow, Water resource management and drought forecasts in South Carolina. J. Am. Water Resour. Assoc. 41 (1) (2005): 145-155.

14. Enterprise risk management-integrated framework, Committee of Sponsoring Organizations of Treadway Commission report, AICPA, 2004.

15. Y. H. Pang and D. J. Shi, Integration of internal control and risk management, in Proc. International conference on business intelligence and financial engineering, (2009): 369-372.

16. L. Coetzee and J. Eksteen, Internet of things - promise for the future? An Introduction, IST Africa 2011, Gaborone, Botswana, 11-13(2011): 9.

17. J. I. Ansell and T. W. Archibald, Data driven risk based management of assets in the water industry, International
Journal of Perform ability Engineering, 4(3) (2008): 215224.

18. R. S. Levine, Data-Driven Operational Risk ManagementRisk, (Amazon publish, 2008).

19. Blackwood and B. Kristen, A data-driven approach to managing operational risk: by identifying, analyzing, and managing operational risks, organizations can undertake new initiatives with greater confidence despite a turbulent economy, The RMA Journal, 91(7) (2009): 68-72.

20. T. Murdoch and M. Cheo, Adopt-A-Stream Foundation. Field Guide: Watershed Inventory and Stream Monitoring Methods, (Everett, WA, 1996).

21. J. H. David and S. M. Barbara, Anomaly detection in streaming environmental sensor data: A data-driven modeling approach, Environmental Modeling \& Software, 25(9) (2010): 1014-1022.

22. M. Morawietz, C. Y. Xu, L. Gottschalk and L. Tallaksen, Systematic evaluation of autoregressive error models as post-processors for a probabilistic streamflow forecast system , Journal of Hydrology , 407(1-4)(2011): 58-72.

23. R. Modarres, Multi-criteria validation of artificial neural network rainfall-runoff modeling, Hydrol. Earth Syst. Sci., 13(2009): 411-421.

24. F. S. Donald, A General Regression Neural Network, IEEE Trans. neural networks, 2(6)(1991): 568-576.

25. E. Koleva and V. Alexandrov, Drought in the Bulgarian low regions during the 20th century, Theoretical and Applied Climatology, 92(1-2) (2008): 113-120.

26. F. Fiorillo and F. M. Guadagno, Karst spring discharges analysis in relation to drought periods, using the SPI, Water Resources Management, 24(9)(2010): 1867-1884.

27. R. C. Eberhart, Y. H. Shi and J. Kennedy , Swarm Intelligence (The Morgan Kaufmann Series in Evolutionary Computation), USA: Academic Press, USA: (Morgan Kaufmann Publishers, 2001), 369-392.

28. W. Wang, Y. S. Ding, R. F. Li and L. J. Cheng, A modified particle swarm optimization algorithm for optimal scheduling of multi-reservoir system, in Proc. 3rd International Conference on Risk Analysis and Crisis Response, ( Laredo, Texas, USA, 2011)

29. R. F. Li, L. J. Cheng, W. Wang and Y. S. Ding, A particle swarm optimization algorithm based collaborative optimal scheduling for multi-level water basin in non-flood season, in Proc. 2011 International Conference on Modeling, Identification and Control, (Shanghai, China, 2011),376381.

30. Drought Monitoring Index for Texas, Technical Report by the Texas A\&M Research Foundation (RF-468511) to the Texas Water Development Board on behalf of the project investigators, 2007.

31. J. Kennedy and R. C. Eberhart, Particle swarm optimization, Proc. IEEE Int'l Conf. on Neural Networks, Piscataway, NJ: IEEE Press, IV, (1995): 1942-1948. 